\title{
Integration analysis for novel IncRNA markers predicting tumor recurrence in human colon adenocarcinoma
}

\author{
Fangyao Chen ${ }^{1}$, Zhe Li ${ }^{2}$, Changyu Deng ${ }^{3}$ and Hong Yan ${ }^{1 *}$ (D)
}

\begin{abstract}
Background: Numerous evidence has suggested that long non-coding RNA (IncRNA) acts an important role in tumor biology. This study focuses on the identification of novel prognostic IncRNA biomarkers predicting tumor recurrence in human colon adenocarcinoma.
\end{abstract}

Methods: We obtained the research data from The Cancer Genome Atlas (TCGA) database. The interaction among different expressed IncRNA, miRNA and mRNA markers between colon adenocarcinoma patients with and without tumor recurrence were verified with miRcode, starBase and miRTarBase databases. We established the IncRNAmiRNA-mRNA competing endogenous RNA (ceRNA) network based on the verified association between the selected markers. We performed the functional enrichment analysis to obtain better understanding of the selected IncRNAs. Then we use multivariate logistic regression to identify the prognostic IncRNA markers with covariates. We also generated a nomogram predicting tumor recurrence risk based on the identified IncRNA biomarkers and clinical covariates.

Results: We included 12,727 IncRNA, 1881 miRNA and 47,761 mRNA profiling and clinical features for 113 colon adenocarcinoma patients obtained from the TCGA database. After filtration, we used 37 specific IncRNAs, 60 miRNAs and 148 mRNAs in the ceRNA network analysis. We identified five IncRNAs as prognostic IncRNA markers predicting tumor recurrence in colon adenocarcinoma, in which four of them were identified for the first time. Finally, we generated a nomogram illustrating the association between the identified IncRNAs and the tumor recurrence risk in colon adenocarcinoma.

Conclusions: The four newly identified IncRNA biomarkers might be potential prognostic biomarkers predicting tumor recurrence in colon adenocarcinoma. We recommend that further clinical and fundamental researches be conducted on the identified IncRNA markers.

Keywords: IncRNA, Colon adenocarcinoma, Tumor recurrence, Integrative analysis, ceRNA network

\section{Background}

Colorectal carcinoma (CRC) is among the most common cancers with high morbidity and mortality among all malignancies and the most common CRC is colon adenocarcinoma (CA) [1]. It was reported that over $70 \%$ of CA patients would develop tumor recurrence within

\footnotetext{
*Correspondence: yanhxjtu@hotmail.com

${ }^{1}$ Department of Epidemiology and Biostatistics, School of Public Health, Xi'an Jiaotong University Health Science Center, 76 Yanta Xilu Road, Xi'an 710061, Shaanxi, China

Full list of author information is available at the end of the article
}

24 months after surgery [2] and tumor recurrence still acts as one of the most severe risk factors to overall survival of CA patients [3]. Thus, the issue of tumor recurrence following a primary $\mathrm{CA}$ becomes very important [4]. It is essential to identify prognostic markers in order to study the biological mechanism in CA and identify the candidate targets for therapy.

Long non-coding RNAs (lncRNAs), with lengths of at least 200 nucleotides, modulate gene expression at the post-transcriptional level [5]. With the innovations in RNA sequencing technologies and computational biology, recent findings suggest that lncRNAs are involved in 
the biological process of cancer development [6]. Numerous studies on the role of lncRNAs in various types of cancers have been performed, and several lncRNA biomarkers have been identified to be related to the development, diagnosis and overall survival of various cancers [7-11]. Recently, lncRNA HOTAIR has been identified to be related to the overall survival in CA [12]. LncRNA $A T B$ is associated with poor prognosis of CRC [13]. LncRNA CCAT1 is reported to be of clinical value in the diagnosis of CA [14]. All these studies suggest a potential value of lncRNAs in the prognosis and diagnosis of CA.

The competing endogenous RNA (ceRNA) hypothesis was presented as a new model demonstrating the association between non-coding and coding RNAs and accepted as one of the most efficient tools in lncRNAs research [10]. It has been widely utilized in the identification of diagnostic and prognostic lncRNA markers in various cancers [15-18].

Few studies have focused on the ceRNA network related to tumor recurrence in CA [19-22]. Thus, in this study, we aim to establish the lncRNA-miRNA-mRNA ceRNA network for the tumor recurrence of CA to identify novel prognostic lncRNA biomarkers for the prediction of tumor recurrence in CA and to achieve better understanding of the role of lncRNAs in CA based on the RNA sequencing data obtained from The Cancer Genome Atlas (TCGA) database.

\section{Materials}

\section{Data profile}

We obtained the RNA sequencing (including miRNA, lncRNA and mRNA) measurements and clinical characteristics of CA patients from The Cancer Genome Atlas (TCGA) database (https://cancergenome.nih.gov/), an open source of information to identify novel biomarkers in cancer research, using R package "TCGAbiolinks" in June 2018. As a result, 12,727 lncRNA, 1881 miRNA and 47,761 mRNA profiling were obtained.

We excluded patients with missing information in tumor recurrence status, tumor location, venous status, lymphatic invasion status, histology type, pathology stage, TNM stage or age at diagnosis. We also excluded records without lncRNA, miRNA or mRNA measurements. Finally, 113 records were included in this study. The maximum follow-up time was 10.37 years (3780 days) with medium follow-up time equal to 1.34 years (488 days). During the follow-up, 6 out of 113 individuals were recorded as deceased.

\section{Data pre-processing}

Since the obtained lncRNA and miRNA expression measurements were not normally distributed, we performed a log transformation to normalize and correct their positively skewed distributions. Suppose $x_{i j}$ is the expression for $j t h$ lncRNA or miRNA expression of $i$ th individual, the transformed value of $j$ th lncRNA or miRNA expression of $i$ th individual would equal to $\ln \left(x_{i j}\right)\left(\right.$ if $x_{i j}>0$ ) or 0 (if $x_{i j}=0$ ). The transformation can be expressed with:

$$
\text { Transformed_expression }=\left\{\begin{array}{ccc}
\ln \left(x_{i j}\right), & \text { if } & x_{i j}>0 \\
0, & \text { if } & x_{i j}=0
\end{array}\right.
$$

Then, before incorporating into multi-variate analysis, to obtain a better explanation to the coefficient obtained in regression analysis, we transform the log-transformed lncRNA expressions into binary variables according to whether the log-transformed expression was higher (upregulated) or lower (down-regulated) than its log-transformed mean.

The mRNA profiling obtained from the TCGA database were normally distributed, therefore, no pre-processing was performed on the mRNA expressions.

\section{Statistical analysis}

All analyses were performed through $\mathrm{R}$ (version 3.4.4, the $\mathrm{R}$ Foundation for Statistical Computing, Vienna, Austria). Clinical and demographic characteristics were tested (with $\alpha=0.05$ ) by Chi-square test (gender, pathology stage and tumor site), Mann-Whitney test (TNM stage) and $t$-test (age at diagnosis). We also used $t$-test to select lncRNAs, miRNAs (log-transformed) and mRNAs with different expression levels between CA patients with and without tumor recurrence (with $\alpha=0.01$ ), the $p$-value obtained were adjusted with the $\mathrm{BH}$ method [23]. Prognostic lncRNA markers were identified based on the adjusted ORs obtained from multi-variate logistic regression.

\section{Establishment of IncRNA-miRNA-mRNA ceRNA network}

We constructed the IncRNA-miRNA-mRNA ceRNA network to identify miRNAs associated mRNAs based on the interaction among lncRNA, miRNA and mRNA that were verified based on the miRcode (http://www.mirco de.org/) [24], starBase (http://starbase.sysu.edu.cn/) [25], and miRTarBase (http://mirtarbase.mbc.nctu.edu.tw/ php/index.php) databases [26]. First, we use $t$-test (with $\mathrm{BH}$ correction) to select lncRNAs, miRNAs and mRNAs with different expression levels between cases with and without tumor recurrence. Then, the differentially expressed lncRNAs, miRNAs and mRNAs, which have been verified in the miRcode, starBase and miRTanBase databases, were incorporated into the construction of lncRNA-miRNA-mRNA ceRNA network. The lncRNAmiRNA-mRNA ceRNA network was conducted using the Cytoscape software (version 3.6.1, National Institute of General Medical Science, Bethesda, MD, US) [27]. We 
also used the "clusterMaker2" [28] application within in the Cytoscape software to identify subnetworks through the Markov Cluster Algorithm (MCL clustering) [28].

\section{Functional annotation analysis}

We performed the functional annotation analysis using "clueGO" [29] application within the Cytoscape software. Gene Ontology (GO) analysis was performed based on the GO database (http://www.geneontology.org/) [30]. Pathway enrichment analysis was performed based on the Kyoto Encyclopedia of Genes and Genomes database (KEGG) (https://www.genome.jp/kegg/pathway.html) [31] and the Reactome (https://www.reactome.org/) databases [32].

\section{Nomogram construction}

Finally, we generated a nomogram predicting tumor recurrence risk for asymptotic CA patient based on the lncRNA markers and clinical features identified in the current study using $\mathrm{R}$ package "rms". The prediction performance was evaluated by the ROC analysis and C-index.

\section{Results}

\section{Characteristics of included patients}

Out of the 113 patients, $50(44.2 \%)$ were males. 67 (59.2\%) patients were diagnosed with stage I/II CA, 46 (40.8\%) were at stage III/IV, 98 (30.9\%). 29 (25.7\%) patients developed venous invasion, 56 (49.6\%) developed lymphatic invasion. The average age at diagnosis for patients with and without tumor recurrence were $71.95 \pm 11.93$ and $65.33 \pm 12.26$ years old. The primary diagnosed sites and detailed TNM stage information of included CA patients are shown in Table 1. Out of the 113 CA patients included in this study, 98 (86.7\%) developed tumor recurrence.

\section{Selection of different expressed RNA sequencing measurements}

We selected the lncRNA, miRNA and mRNA measurements with different expression levels between patients with and without tumor recurrence through independent $t$-tests with $p$-value adjusted through the BH method [19]. As a result, we selected 61 lncRNA, 167 miRNA and 354 mRNA measurements with adjusted $p$-value less than 0.01 . The selected lncRNAs, miRNAs and mRNAs are contained in Additional file 1: S1 (sheets 1-3).

\section{Establishment of IncRNA-miRNA-mRNA ceRNA network}

We used the miRcode, starBase and miRTarBase databases to verify the interaction relationship between the different expressed lncRNA, miRNA and mRNA markers. Based on the verified interaction relationship, we
Table 1 Clinical characteristics of included CA patients

\begin{tabular}{|c|c|c|c|c|c|}
\hline \multirow[t]{2}{*}{ Factor } & \multirow[t]{2}{*}{ Categories } & \multicolumn{2}{|c|}{ Tumor status } & \multirow[t]{2}{*}{ Total } & \multirow[t]{2}{*}{$p$-value } \\
\hline & & Free & Recurred & & \\
\hline \multirow[t]{2}{*}{ Gender } & Female & 10 & 53 & 63 & $0.361^{a}$ \\
\hline & Male & 5 & 45 & 50 & \\
\hline \multirow[t]{2}{*}{ Venous invasion } & No & 11 & 73 & 84 & $0.924^{\mathrm{a}}$ \\
\hline & Yes & 4 & 25 & 29 & \\
\hline \multirow{2}{*}{$\begin{array}{l}\text { Lymphatic inva- } \\
\text { sion }\end{array}$} & No & 9 & 48 & 57 & $0.427^{a}$ \\
\hline & Yes & 6 & 50 & 56 & \\
\hline \multirow[t]{2}{*}{ Pathology stage } & $|/| \mid$ & 10 & 57 & 67 & $0.053^{\mathrm{a}}$ \\
\hline & III/IV & 5 & 41 & 46 & \\
\hline \multirow[t]{5}{*}{ Tstage } & $\mathrm{T} 1$ & 0 & 3 & 3 & $0.151^{b}$ \\
\hline & $\mathrm{T} 2$ & 2 & 19 & 21 & \\
\hline & $\mathrm{T} 3$ & 9 & 64 & 73 & \\
\hline & $\mathrm{T} 4$ & 4 & 12 & 16 & \\
\hline & NO & 10 & 58 & 68 & $0.295^{b}$ \\
\hline \multirow[t]{2}{*}{ N stage } & $\mathrm{N} 1$ & 4 & 19 & 23 & \\
\hline & N2 & 1 & 21 & 22 & \\
\hline \multirow[t]{2}{*}{ M stage } & MO & 14 & 81 & 95 & $0.293^{\mathrm{a}}$ \\
\hline & M1 & 1 & 17 & 18 & \\
\hline \multirow[t]{5}{*}{ Tumor site } & Ascending colon & 4 & 20 & 24 & $0.009^{a}$ \\
\hline & Cecum & 7 & 18 & 25 & \\
\hline & Sigmoid colon & 1 & 42 & 43 & \\
\hline & Transverse colon & 3 & 7 & 10 & \\
\hline & Other ${ }^{c}$ & 0 & 11 & 11 & \\
\hline \multirow[t]{2}{*}{ Age at diagnosis } & Mean & 65.33 & 71.95 & - & $0.049^{d}$ \\
\hline & SD & 12.26 & 11.93 & - & \\
\hline
\end{tabular}

${ }^{a}$ With Chi-square test

b With Mann-Whitney test

c Including descending colon, hepatic flexure and splenic flexure

${ }^{d}$ With t-test

conducted the IncRNA-miRNA-mRNA ceRNA network analysis to reveal the association between selected lncRNAs and miRNAs as shown in Fig. 1. The detailed information is shown in Tables 2 and 3. As a result, the ceRNA network indicated that 60 particular miRNAs interacted with 33 specific lncRNAs and 148 mRNAs.

\section{Functional enrichment analysis}

In order to obtain a deep understanding on the selected genes, we performed the functional enrichment analysis to the intersection mRNAs as shown in Fig. 2. It revealed that the enriched GO terms for biological process (BP) were mainly related to the several immune processes including the regulation of the differentiation and proliferation for several immune cells ( $\mathrm{T}$ cell, mononuclear cell and leukocyte), the regulation of cytotoxicity and immunity mediated by natural killer cell and leukocyte, the differentiation of dendritic cell, the regulation of interleukin-10 and -12 and the regulation of cytokine, as shown in Fig. 2a. 


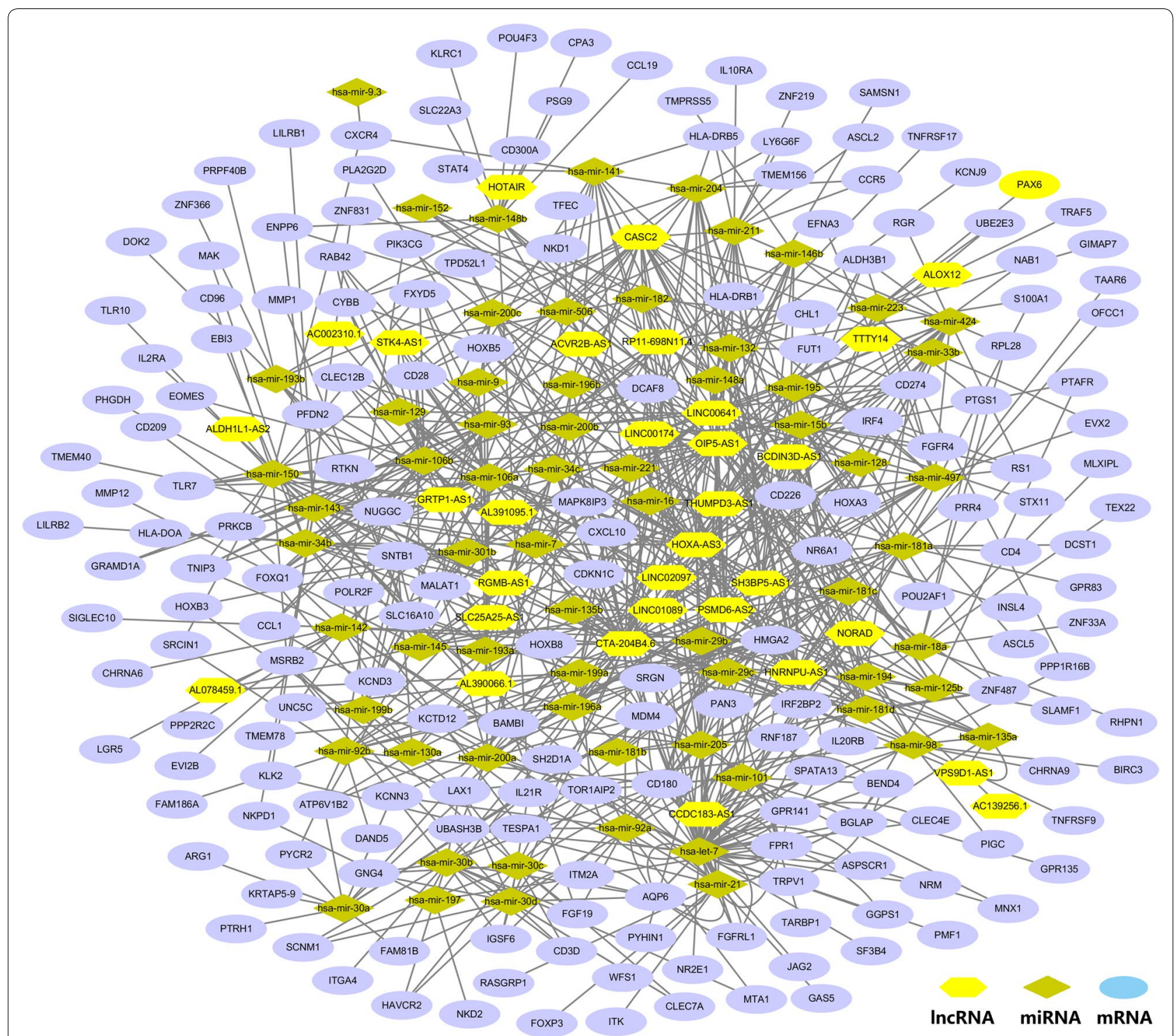

Fig. 1 The IncRNA-miRNA-mRNA ceRNA network. The hexagon represents IncRNAs, the circle stands for the mRNAs and the diamond is for the miRNAs

The associated cell component $(\mathrm{CC})$ included protein phosphatase type 2A complex and mast cell granule as shown in Fig. 2b. The enriched GO terms for molecular function (MF) were mainly about core promoter binding, interleukin-10 activity, MHC class 1 activity, as shown in Fig. 2c.

The pathways enriched based on the KEGG and Reactome databases are shown in Fig. 2d. The revealed pathways included RHO GTPases Activate Rhotekin and Rhophilins pathway, Interleukin-21 signaling pathway, Interleukin-2 family signaling pathway and Nuclear Receptor transcription pathway.

\section{Subnetwork analysis}

We used the "clusterMaker2" [28] application within the Cytoscape software to identify the subnetworks from the main ceRNA network through the MCL clustering approach [28]. As a result, we identified 37 distinct subnetworks. Based on the number of nodes in each subnetworks, after filtration, we selected 4 subnetworks with at least 10 nodes, as shown in Fig. 3. We also performed the functional enrichment analysis for the genes in the selected subnetworks; the results are shown in Fig. 4. The enriched GO terms for BP in subnetwork 1 and 4 were mainly about several human 
Table 2 IncRNA targeted miRNA verified in CA

IncRNA Targeted miRNA

AC002310.1

hsa-mir-129

AC139256.1

ACVR2B-AS1 hsa-mir-125b, hsa-mir-141, hsa-mir-143, hsa-mir-182, hsa-mir-200a, hsa-mir-7

AL078459.1 hsa-mir-145, hsa-mir-199a, hsa-mir-7

AL390066.1 hsa-mir-106a, hsa-mir-141, hsa-mir-150, hsa-mir-200a, hsa-mir-205, hsa-mir-9, hsa-mir-93, hsa-mir-145, hsa-mir-29b, hsa-mir-29c

AL391095.1 hsa-mir-106a, hsa-mir-141, hsa-mir-150, hsa-mir-200a, hsa-mir-205, hsa-mir-9, hsa-mir-93, hsa-mir-145, hsa-mir-29b, hsa-mir-29c

ALDH1L1-AS2 hsa-mir-145

BCDIN3D-AS1 hsa-mir-125b, hsa-mir-146b, hsa-mir-182, hsa-mir-18a, hsa-mir-205

CASC2

hsa-mir-101, hsa-mir-106a, hsa-mir-125b, hsa-mir-141, hsa-mir-143, hsa-mir-15b, hsa-mir-16, hsa-mir-181a, hsa-mir-181c, hsa-mir-181d, hsa-mir-193a, hsa-mir-193b, hsa-mir-194, hsa-mir-195, hsa-mir-200a, hsa-mir-200b, hsa-mir-200c, hsa-mir-204, hsa-mir-205, hsa-mir-21, hsa-mir-211, hsa-mir-33b, hsa-mir-34b, hsa-mir-34c, hsa-mir-424, hsa-mir-497, hsa-mir-506, hsa-mir-93

CCDC183-AS1 hsa-let-7, hsa-mir-205, hsa-mir-205, hsa-mir-29b, hsa-mir-29c, hsa-mir-98

CTA-204B4.6 hsa-let-7, hsa-mir-106a, hsa-mir-106b, hsa-mir-128, hsa-mir-129, hsa-mir-130a, hsa-mir-132, hsa-mir-135a, hsa-mir-135b, hsa-mir-142, hsa-mir-143, hsa-mir-145, hsa-mir-146b, hsa-mir-150, hsa-mir-15b, hsa-mir-16, hsa-mir-181a, hsa-mir-181c, hsa-mir-181d, hsa-mir-195, hsa-mir-196a, hsa-mir-196b, hsa-mir-197, hsa-mir-200b, hsa-mir-200c, hsa-mir-204, hsa-mir-205, hsa-mir-211, hsa-mir-221, hsa-mir-29b, hsa-mir-29c, hsa-mir-301b, hsa-mir-30a, hsa-mir-30b, hsa-mir-30c, hsa-mir-30d, hsa-mir-33b, hsa-mir-34b, hsa-mir-34c, hsa-mir-424, hsamir-497, hsa-mir-92a, hsa-mir-92b, hsa-mir-93, hsa-mir-98

GRTP1-AS1 hsa-mir-106a, hsa-mir-106b, hsa-mir-146b, hsa-mir-150, hsa-mir-93

HNRNPU-AS1 hsa-mir-125b, hsa-mir-129, hsa-mir-132, hsa-mir-135a, hsa-mir-135b, hsa-mir-145, hsa-mir-145, hsa-mir-15b, hsa-mir-16, hsa-mir-181a, hsa-mir-181c, hsa-mir-181d, hsa-mir-18a, hsa-mir-195, hsa-mir-199a, hsa-mir-204, hsa-mir-205, hsa-mir-211, hsa-mir-424, hsa-mir-497, hsa-mir-506

HOXA-AS3 hsa-mir-106a, hsa-mir-106a, hsa-mir-106b, hsa-mir-125b, hsa-mir-132, hsa-mir-141, hsa-mir-143, hsa-mir-150, hsa-mir-15b, hsa-mir-16, hsa-mir-18a, hsa-mir-195, hsa-mir-200a, hsa-mir-204, hsa-mir-205, hsa-mir-211, hsa-mir-29b, hsa-mir-29c, hsa-mir-34b, hsa-mir-34c, hsamir-424, hsa-mir-497, hsa-mir-506, hsa-mir-7, hsa-mir-9, hsa-mir-93

HOTAIR hsa-mir-148b

LINC00174 hsa-mir-130a, hsa-mir-15b, hsa-mir-16, hsa-mir-195, hsa-mir-199a, hsa-mir-204, hsa-mir-211, hsa-mir-301b, hsa-mir-424, hsa-mir-497

LINC00641 hsa-mir-129, hsa-mir-132, hsa-mir-135a, hsa-mir-135b, hsa-mir-145, hsa-mir-146b, hsa-mir-148a, hsa-mir-148b, hsa-mir-152, hsa-mir-15b, hsa-mir-16, hsa-mir-195, hsa-mir-200b, hsa-mir-200c, hsa-mir-204, hsa-mir-211, hsa-mir-221, hsa-mir-424, hsa-mir-497, hsa-mir-506, hsamir-7

LINC01089 hsa-let-7, hsa-mir-128, hsa-mir-129, hsa-mir-135a, hsa-mir-135b, hsa-mir-145, hsa-mir-15b, hsa-mir-16, hsa-mir-193a, hsa-mir-193b, hsamir-195, hsa-mir-34b, hsa-mir-34c, hsa-mir-424, hsa-mir-497, hsa-mir-98

LINC02097 hsa-mir-125b, hsa-mir-128, hsa-mir-143, hsa-mir-33b

NORAD hsa-let-7, hsa-mir-101, hsa-mir-181a, hsa-mir-181c, hsa-mir-181d, hsa-mir-182, hsa-mir-194, hsa-mir-199a, hsa-mir-204, hsa-mir-205, hsamir-211, hsa-mir-92a, hsa-mir-92b, hsa-mir-98

OIP5-AS1 hsa-let-7, hsa-mir-130a, hsa-mir-132, hsa-mir-141, hsa-mir-143, hsa-mir-145, hsa-mir-146b, hsa-mir-148a, hsa-mir-148b, hsa-mir-150, hsa-mir-152, hsa-mir-15b, hsa-mir-16, hsa-mir-181a, hsa-mir-181c, hsa-mir-181d, hsa-mir-18a, hsa-mir-194, hsa-mir-195, hsa-mir-196a, hsa-mir-196b, hsa-mir-200a, hsa-mir-200b, hsa-mir-200c, hsa-mir-205, hsa-mir-221, hsa-mir-223, hsa-mir-301b, hsa-mir-424, hsa-mir-497, hsa-mir-7, hsa-mir-98

PSMD6-AS2 hsa-mir-106a, hsa-mir-135a, hsa-mir-135b, hsa-mir-141, hsa-mir-148a, hsa-mir-148b, hsa-mir-181a, hsa-mir-181c, hsa-mir-181d, hsa-mir-182, hsa-mir-200a, hsa-mir-204, hsa-mir-211, hsa-mir-9, hsa-mir-93

RGMB-AS1 hsa-mir-129, hsa-mir-143, hsa-mir-150, hsa-mir-34c, hsa-mir-7, hsa-mir-92a, hsa-mir-92b

RP11-698N11.4 hsa-mir-106a, hsa-mir-128, hsa-mir-132, hsa-mir-142, hsa-mir-148a, hsa-mir-148b, hsa-mir-152, hsa-mir-223, hsa-mir-34b, hsa-mir-34c, hsamir-7

SH3BP5-AS1 hsa-mir-101, hsa-mir-106a, hsa-mir-125b, hsa-mir-132, hsa-mir-143, hsa-mir-146b, hsa-mir-15b, hsa-mir-16, hsa-mir-181a, hsa-mir-181C, hsa-mir-181d, hsa-mir-182, hsa-mir-195, hsa-mir-199a, hsa-mir-200a, hsa-mir-204, hsa-mir-211, hsa-mir-223, hsa-mir-34b, hsa-mir-34c, hsa-mir-424, hsa-mir-497, hsa-mir-93

SLC25A25-AS1 hsa-mir-150, hsa-mir-193a, hsa-mir-193b, hsa-mir-199a, hsa-mir-205, hsa-mir-34b, hsa-mir-34c, hsa-mir-9

STK4-AS1 hsa-mir-106a, hsa-mir-106a, hsa-mir-106b, hsa-mir-182, hsa-mir-93

THUMPD3-AS1 hsa-mir-143, hsa-mir-145, hsa-mir-146b, hsa-mir-15b, hsa-mir-16, hsa-mir-181a, hsa-mir-181c, hsa-mir-181d, hsa-mir-195, hsa-mir-221, hsamir-29b, hsa-mir-29c, hsa-mir-424, hsa-mir-497, hsa-mir-9

TTTY14 hsa-mir-33b

VPS9D1-AS1 hsa-mir-135a, hsa-mir-135a, hsa-mir-135b 
Table 3 miRNA targeted mRNA verified in miRTarBase databases

\begin{tabular}{|c|c|}
\hline miRNA & Targeted mRNA \\
\hline hsa-let-7 & $\begin{array}{l}\text { AQP6, ASPSCR1, BEND4, BGLAP, CLEC4E, DCAF8, FGFRL1, FPR1, GGPS1, GPR141, HMGA2, IRF2BP2, JAG2, KCTD12, MDM4, } \\
\text { NR2E1, NR6A1, NRM, PMF1, PPPIR16B, RS1, SF3B4, SPATA13, TOR1AIP2, TOR1AIP2, TRPV1, UBASH3B }\end{array}$ \\
\hline hsa-mir-101 & BEND4, CD180, CD180, GPR135, IL20RB, MNX1, TOR1AIP2 \\
\hline hsa-mir-106a & CD28, CLEC12B, DCAF8, FOXQ1, FXYD5, HMGA2, KCND3, NUGGC, PRKCB, RAB42, RAB42, TLR7, TNIP3 \\
\hline hsa-mir-106b & BAMBI, CCL1, CD28, CLEC12B, CYBB, DCAF8, EOMES, FOXQ1, FXYD5, GRAMD1A, KCND3, NUGGC, PRKCB, RAB42, TLR7, TNIP3 \\
\hline hsa-mir-125b & BGLAP, PTAFR \\
\hline hsa-mir-128 & $A S C L 5, C Y B B, I L 21 R$ \\
\hline hsa-mir-129 & HOXB8 \\
\hline hsa-mir-130a & ATP6V1B2, FOXQ1, HOXB3, SNTB1 \\
\hline hsa-mir-132 & CD226, CHL1, FUT1, KCNJ9, S100A1, SAMSN1, TNFRSF17 \\
\hline hsa-mir-135a & $\angle A X 1, B G L A P$ \\
\hline hsa-mir-141 & HLA-DRB1, HLA-DRB5, HOXB5, MALAT1, MDM4, STAT4 \\
\hline hsa-mir-142 & CHRNA6, EVI2B, HMGA2, KCND3, KCTD12, LGR5, PIK3CG, PPP2R2C, PRKCB, SH2D1A, SIGLEC10, TNIP3, TOR1AIP2 \\
\hline hsa-mir-143 & BAMBI, ENPP6, IL2RA, KLK2, LILRB1, MAPK8IP3, NKPD1, PHGDH, SLC16A10, TMEM40 \\
\hline hsa-mir-145 & CD28, HMGA2, MMP1, MMP12, RTKN, SLC16A10, SNTB1 \\
\hline hsa-mir-146b & MALAT1, PTGS1, TMPRSS5 \\
\hline hsa-mir-148a & HLA-DRB1, PAN3 \\
\hline hsa-mir-148b & CCL19, CD300A, CPA3, CYBB, ENPP6, IL21R, KLRC1, PIK3CG, POU4F3, PSG9, SLC22A3 \\
\hline hsa-mir-150 & CD96, CXCR4, FXYD5, MSRB2, NKD1, NUGGC, SRCIN1, TLR10, TLR7, TNIP3 \\
\hline hsa-mir-152 & CD274 \\
\hline hsa-mir-15b & ALDH3B1, BAMBI, CD180, CD274, FGFR4, HOXA3, IL20RB, IRF4, NAB1, NR6A1, POU2AF1, RS1, STX11 \\
\hline hsa-mir-16 & CD226, HMGA2, INSL4 \\
\hline hsa-mir-181a & CD4, CHL1, DCST1, GPR83, HMGA2, NR6A1, OFCC1, PRR4, RNF187, S100A1, SRGN, TAAR6, ZNF487 \\
\hline hsa-mir-181b & HMGA2, NR6A1, RNF187, SH2D1A, SRGN, ZNF487 \\
\hline hsa-mir-181c & HMGA2, KCNN3, NR6A1, RNF187, SRGN, ZNF487 \\
\hline hsa-mir-181d & HMGA2, NR6A1, RNF187, SRGN, ZNF487 \\
\hline hsa-mir-182 & CHL1, ZNF831 \\
\hline hsa-mir-18a & DCAF8, MLXIPL, PTGS1, RHPN1, RNF187, RPL28, TEX22, ZNF33A \\
\hline hsa-mir-193a & FGF19, RTKN \\
\hline hsa-mir-193b & DOK2, FXYD5, PFDN2, PRPF4OB, RTKN, ZNF366 \\
\hline hsa-mir-194 & AQP6, CD274, HMGA2, MAPK8IP3, SLAMF1, SPATA13 \\
\hline hsa-mir-195 & ALDH3B1, ALOX12, CD180, CD274, FGFR4, HOXA3, IRF4, NKD1, NR6A1, POU2AF1, RS1, UBE2E3 \\
\hline hsa-mir-196a & ATP6V1B2, CXCL10, FUT1, HMGA2, HOXB8, NR6A1, TMEM78, TRPV1 \\
\hline hsa-mir-196b & HMGA2, HOXB8, NR6A1, PIK3CG \\
\hline hsa-mir-197 & FPR1, NKD2, NKPD1 \\
\hline hsa-mir-199a & CDKN1C, LAX1, POLR2F, SLC16A10, SNTB1, TOR1AIP2, UNC5C \\
\hline hsa-mir-199b & LAX1, POLR2F, SLC16A10, SNTB1, TOR1AIP2, UNC5C \\
\hline hsa-mir-200a & HOXB5, MALAT1, MDM4, MNX1, UBASH3B \\
\hline hsa-mir-200b & CD274, HOXB5, MALAT1, MDM4, TPD52L1 \\
\hline hsa-mir-200c & CXCL10, HOXB5, MALAT1, POLR2F, TPD52L1 \\
\hline hsa-mir-204 & CCR5, CD28, CXCR4, CYBB, HLA-DRB1, HLA-DRB5, HMGA2, IRF2BP2, LY6G6F, MALAT1, TMEM156 \\
\hline hsa-mir-205 & BAMBI, GPR141, IGSF6, ITM2A, PYHIN1 \\
\hline hsa-mir-21 & CCL1, CXCL10, DCAF8, FGFRL1, FOXP3, GAS5, ITK, KLK2, MDM4, PAN3, RASGRP1, SLC16A10, TOR1AIP2, WFS1 \\
\hline hsa-mir-211 & ASCL2, CCR5, CD28, HLA-DRB1, HLA-DRB5, IL1ORA, IRF2BP2, LY6G6F, RPL28, TMEM156, ZNF219 \\
\hline hsa-mir-221 & CDKN1C, HOXB5, PTAFR \\
\hline hsa-mir-223 & CD226, GIMAP7, PAX6, PPP1R16B, TRAF5, UBE2E3 \\
\hline hsa-mir-29b & IRF2BP2, KCTD12 \\
\hline hsa-mir-29c & CD274, IRF2BP2, TARBP1, TESPA1 \\
\hline hsa-mir-301b & ATP6V1B2, CLEC12B, FOXQ1, HLA-DOA, HOXB3, LILRB2, SNTB1 \\
\hline hsa-mir-30a & ARG1, ATP6V1B2, BAMBI, FAM81B, HMGA2, IL21R, KCNN3, KRTAP5-9, MDM4, PTRH1, PYCR2 \\
\hline
\end{tabular}


Table 3 (continued)

\begin{tabular}{ll}
\hline miRNA & Targeted mRNA \\
\hline hsa-mir-30b & AQP6, CD180, CD3D, CLEC7A, FAM81B, FGF19, GNG4, HAVCR2, IGSF6, IL21R, ITGA4, LAX1, MSRB2, MTA1, SCNM1, TOR1AIP2 \\
hsa-mir-30c & AQP6, CD180, CD3D, CLEC7A, FGF19, GNG4, HAVCR2, IGSF6, LAX1, MSRB2, SCNM1, TOR1AIP2 \\
hsa-mir-30d & AQP6, AQP6, CD3D, CLEC7A, FAM81B, GNG4, HAVCR2, IGSF6, IL21R, LAX1, MSRB2, RNF187, SCNM1, TOR1AIP2 \\
hsa-mir-33b & EVX2, HMGA2 \\
hsa-mir-34b & CD209, CD274, EBI3, MAK, POLR2F, TESPA1, ZNF831 \\
hsa-mir-34c & MDM4, PLA2G2D \\
hsa-mir-424 & ALDH3B1, CD180, CD274, FGFR4, HOXA3, NR6A1, RGR, RS1 \\
hsa-mir-497 & ALDH3B1, CD180, CD274, EFNA3, EVX2, FGFR4, HOXA3, IRF4, NR6A1, RS1 \\
hsa-mir-506 & ENPP6, FOXQ1, HMGA2, TFEC \\
hsa-mir-7 & MDM4, PFDN2, SPATA13 \\
hsa-mir-9.3 & $C X C R 4$ \\
hsa-mir-92a & FGF19, KCNN3, MTA1, TESPA1 \\
hsa-mir-92b & $C D 180$, CD226, CDKN1C, DAND5, FAM186A, HMGA2, LAX1, NUGGC, PYCR2, SRCIN1 \\
hsa-mir-93 & $C C L 1$, CD28, CLEC12B, DCAF8, FOXQ1, FXYD5, GRAMD1A, KCND3, NUGGC, PFDN2, PRKCB, RAB42, TLR7, TNIP3, UNC5C \\
hsa-mir-98 & BIRC3, CHRNA9, HMGA2, PIGC, TNFRSF9 \\
\hline
\end{tabular}

immune processes, which consistent with that obtained from the main network

\section{Identification of prognostic IncRNA markers}

We applied the univariate logistic regression model to estimate the ORs for patients with different expression types of the each lncRNA markers in developing tumor recurrence or not separately. Among the 39 lncRNAs identified in ceRNA network, 8 lncRNAs yielded statistical significance $(p<0.05)$ in univariate logistic regression and were incorporated into multivariate logistic regression with clinical covariates.

Finally, 5 lncRNAs (CASC2, AL078459.1, AL390066.1, STK4-AS1 and HOXA-AS3) yielded statistical significance $(p<0.05)$ in multivariate logistic analysis with age at diagnosis and tumor pathology stage as covariates. The result suggests that these lncRNA markers might act as prognostic predictors for the tumor recurrence in CA. The adjusted ORs of the 5 identified prognostic IncRNAs, in particular, indicated that the up-regulation of CASC2 $(O R=1.225,95 \% \mathrm{CI} 1.061-$ 5.025), AL078459.1 (OR=2.923, 95\% CI 1.504-7.946), $A L 390066.1,(O R=2.311,95 \%$ CI 1.182-4.764) STK4AS1 (OR $=3.611,95 \%$ CI 1.328-3.030) and HOXA-AS3 $(O R=2.511,95 \%$ CI 1.026-4.415) may be associated with the development of tumor recurrence of $\mathrm{CA}$.

\section{Nomogram for tumor recurrence prediction}

Finally, we generated a simple-to-use nomogram based on the 5 prognostic lncRNA markers and clinical characteristics (pathology stage and age at diagnosis) of CA patients as shown in Fig. 5. It could provide useful information in prediction of tumor recurrence for asymptomatic CA patients based on multivariate logistic regression. The $\mathrm{C}$-index for the model was 0.895 and the area under the ROC for the model is 0.885 (95\% CI based on bootstrap method: 0.836-0.935). Both C-index and the ROC analysis suggested a good predict performance.

\section{Discussion}

\section{General comments}

Differential expression of lncRNAs has been widely identified in various cancers. Published studies have revealed that lncRNAs have key roles in vital biological functions of cancers. However, only few studies have described the role of lncRNA profiles in tumor recurrence of CA [19-22]. In this study, we focused on the identification of novel prognostic markers for the tumor recurrence of CA based on the RNA sequencing data from the TCGA database. We have constructed the IncRNA-miRNA-mRNA ceRNA network to clarify the unknown ceRNA regulatory network in tumor recurrence of $\mathrm{CA}$. As a result, 5 lncRNAs (CASC2, AL078459.1, AL390066.1, STK4-AS1 and $H O X A-A S 3)$ were identified through the ceRNA network and multivariate logistic regression. We also performed functional enrichment analysis to investigate the molecular role of the identified lncRNA biomarkers.

For the 5 identified lncRNA markers, CASC2 has been reported to be associated with colorectal cancer [33-35]. Studies have revealed that the deregulation of CASC2 by miRNA hsa-mir-21 and hsa-mir-18a increases the proliferation and migration of cancer cells in colorectal cancer [33-35]. The link between the CASC2 and the prognosis of CA suggested in published literatures were consistent with the results in this study. This also indicated that the results of our research were 


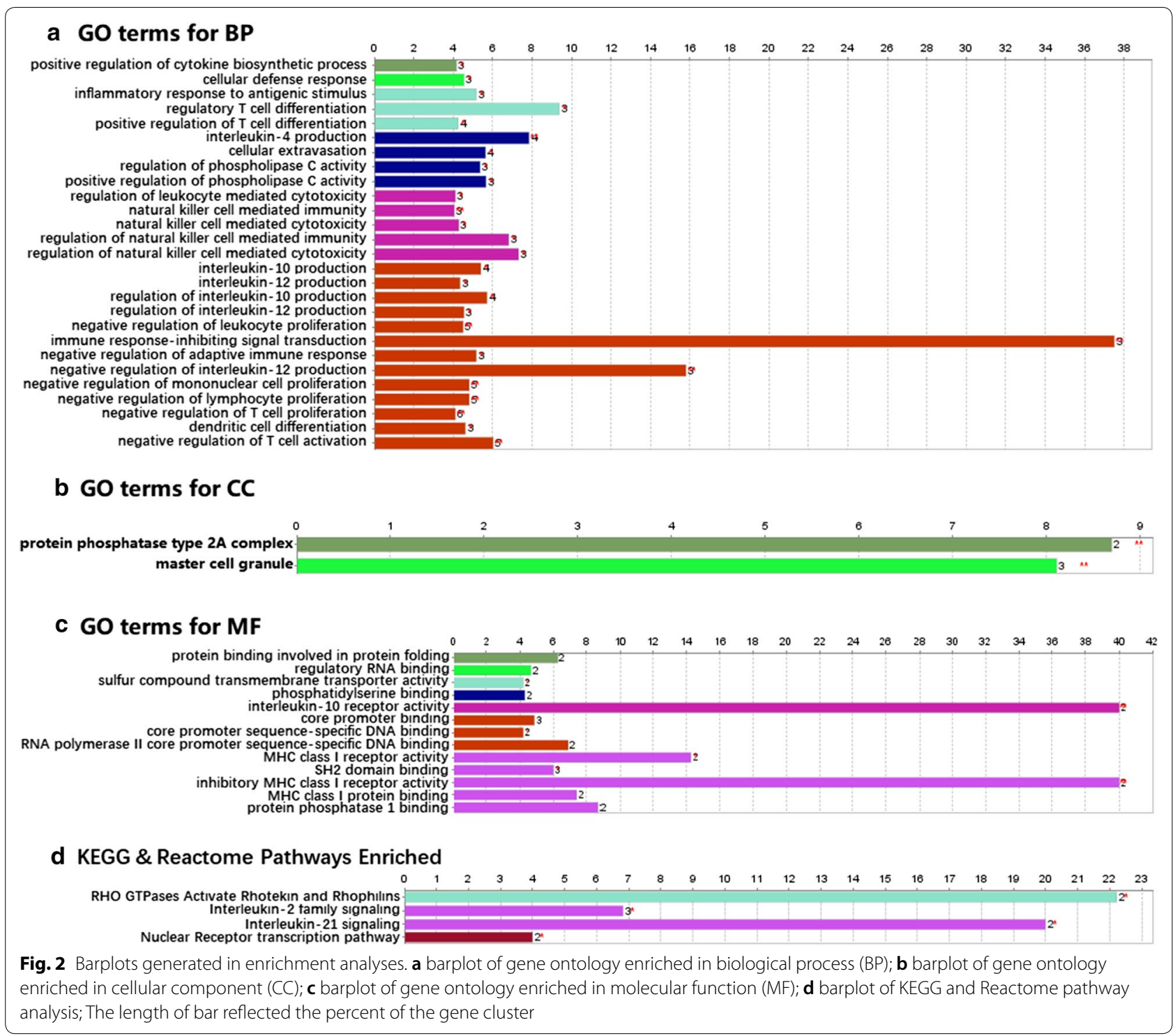

reasonable. For $S T K 4-A S 1$, it was associated with protein coding gene $S T K 4$ while the down-regulation of STK4 was associated with the invasion and migration of colorectal cancer [36]. This also suggests that the association between STK4-AS1 and the prognosis of CA revealed in this study was reasonable. For HOXA-AS3, though it has not been identified in CA before, the upregulation of HOXA-AS3 was reported to be associated with tumor progression and poor prognosis in glioma [37]. In this study, we found STK4-AS1 and HOXA-AS3 were also related to tumor recurrence in CA. For lncRNAs AL078459.1 and AL390066.1, though no functional roles have been reported in CA before this work, in our study, have been identified to be related to the tumor recurrence in CA.
To obtain a deep understanding of the selected lncRNA markers, the functional enrichment analyses were performed. The enriched in GO terms were related with several human immune process. IL-10 and IL-12, as representative immune factors, play an important role in inflammation and tumorigenesis [38], and published research also suggested a potential relationship between them and the progression of CRC [39]. Tumor infiltrating T-cells was related to the microsatellite instability and the prognosis of CRC [40]. Natural killer cell plays an important role in the anti-cancer defense, and has great potential in cancer immunotherapy in cancer immunotherapy [41]. Dendritic cell [42], tumor infiltrating mononuclear cell [43] and tumor infiltrating mast cell [44] were all associated with the progression and prognosis of CRC. 


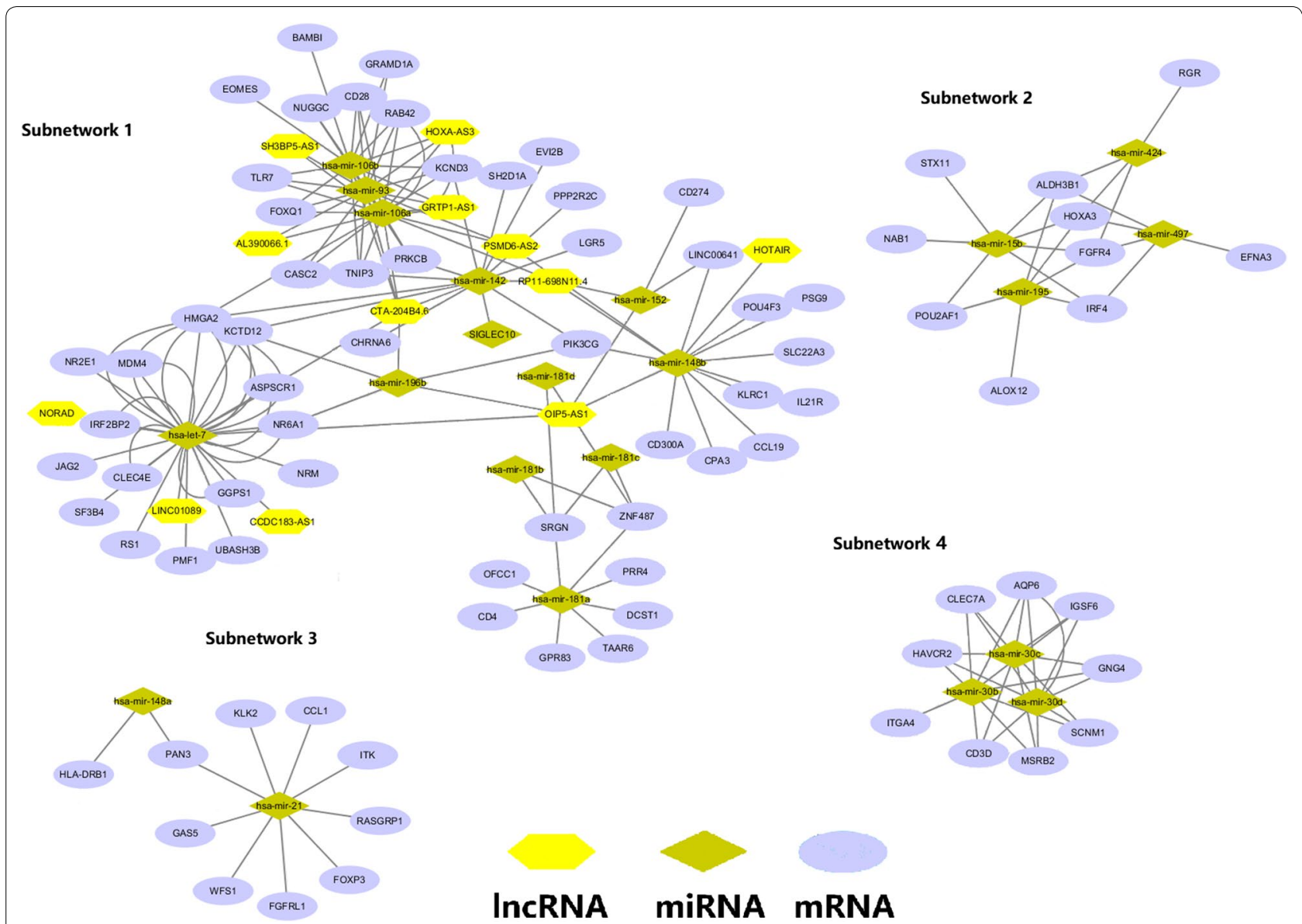

Fig. 3 Subnetwork analysis based on the main ceRNA network. A total of 37 subnetworks were identified while only subnetworks with at least 10 nodes (subnetwork 1 to 4) were selected. The hexagon represents IncRNAs, the circle stands for the mRNAs and the diamond is for the miRNAs

MHC class I is a major component of tumor-associated antigen presenting system, which responded to a large number of chemotherapeutic agents in the treatment of CRC [45]. For the enriched pathways, RHO GTPases Activate Rhotekin and Rhophilins pathway was associated with the development and progression of several solid malignancies including CRC [46, 47], Interleukin-21 signaling pathway was associated with the development of colitis-associated CRC [48] and Interleukin-2 family signaling pathway acts important role in current antitumor immunotherapy [49]. The immune infiltration is closely associated with prognosis of CRC $[50,51]$, the results of enrichment analysis suggested that the genes in the ceRNA network were associated with several important human immune processes, thus may be associated with the clinical outcome of CRC.

For the subnetwork analysis, we identified 37 distinct subnetworks while only those with at least 10 nodes were selected (as shown in Fig. 3). Then, we performed the functional enrichment analysis for the genes involved in each subnetworks. For subnetwork 1, the enriched GO terms for BP were mainly about the co-stimulation of $\mathrm{T}$ cell and lymphocyte cell, the differentiation of endodermal cell and the proliferation of stem cell. For subnetwork 3, the enriched biological processes were mainly about the regulation of IL-17 and IL4, the differentiation of thymus and the regulation of the production of interferon gamma, all related to the progression and prognosis of CA [52-55]. Most of biological processes enriched for the subnetworks were about human immune process and this was consistent with the biological processes enriched for the main network. For the enriched terms for MF in subnetwork 1, though different from that enriched from the main network, phosphatidylcholine has been found to involve in the growth of CRC cell [56]. The enriched pathways based on the KEGG and Reactome databases for subnetwork 2 (nuclear transcript pathway) were consistent with those enriched in the main network. The consistency in functional enrichments between the subnetworks and the main network suggested good robustness of our analysis. 


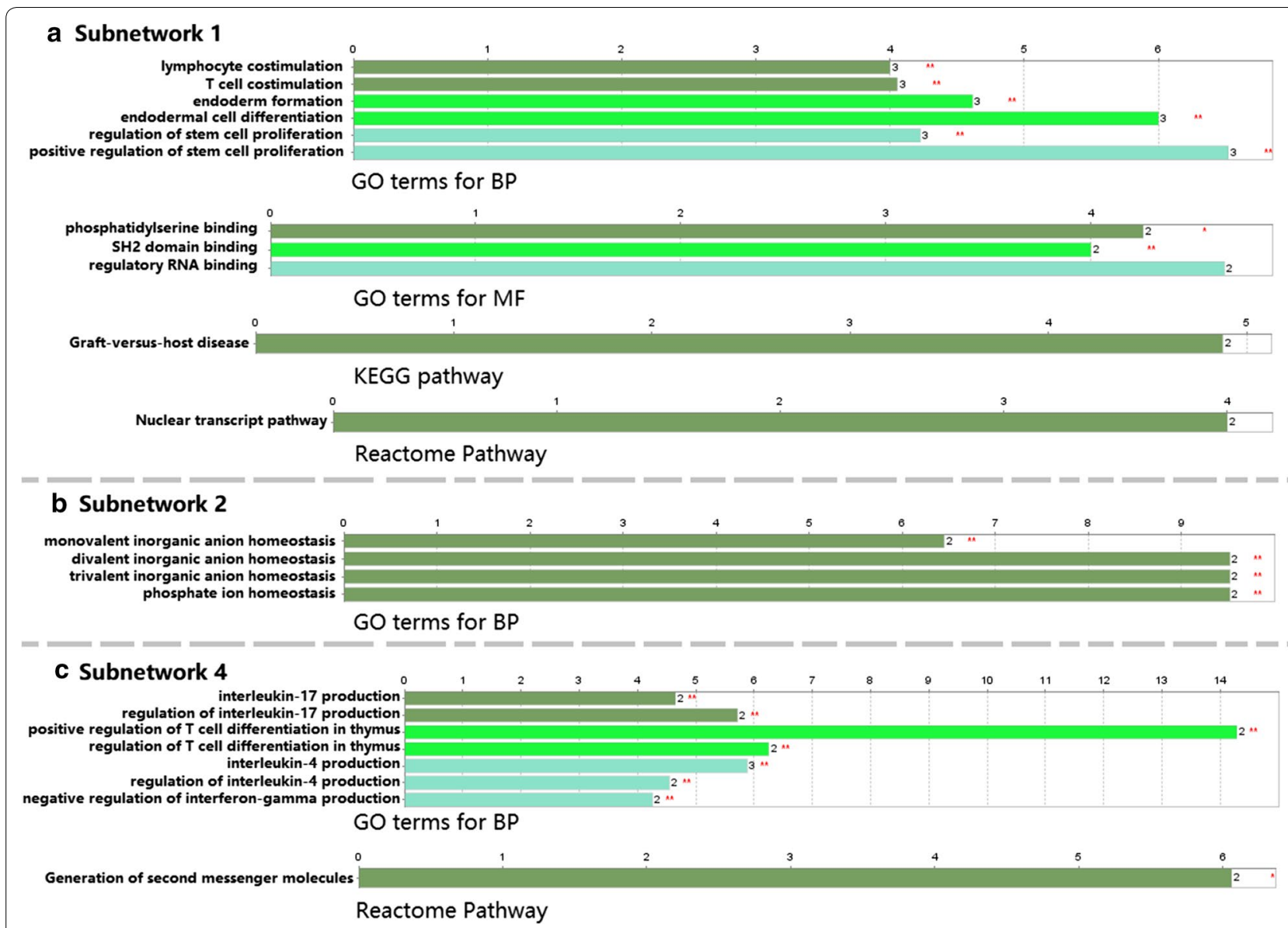

Fig. 4 Functional enrichments for the genes in the subnetworks. a Enriched GO terms for biological process (BP), molecular function (MF) and pathways for subnetwork 1; b enriched GO terms for biological process (BP) for subnetwork 2; c enriched GO terms for biological process (BP), and pathway for subnetwork 4

The nomogram generated in this study was simpleto-use and would be useful in estimating the tumor recurrence risk for asymptotic patients with CA. It also visualized the associations between each prognostic lncRNA and clinical features (stage and age at diagnosis) and the tumor prognosis of CA patients.

Recently, many studies on the identification of prognostic genes in CA based on the TCGA database have been reported and their findings are then verified with functional experiments in succession [57-60]. Those studies all suggested a good reliability of the TCGA database in identifying new prognostic gene signatures for the prognosis in cancer studies and the potential value of the results obtained in the current work.

\section{Limitations}

Firstly, our study was a preliminary work. In this study, we obtained the RNA sequencing and clinical data from public database, but no clinical samples were involved. Thus, the importance of the selected markers still need to be validated in future cohort study. Then, though we tried to incorporated as many clinical factors as possible in our analysis, some important factors were still not available, for example, treatment information (such as chemotherapy, surgery and radiotherapy), and living habits (such as smoking or drinking habits). These might cause potential bias in analysis.

\section{Conclusion}

In this study, we identified five prognostic lncRNA markers for the prediction of tumor recurrence in CA based on ceRNA hypothesis and data obtained from the TCGA database, in which, four of the selected lncRNA markers (AL078459.1, AL390066.1, STK4-AS1 and $H O X A-A S 3)$ were identified for the first time. The hub genes in the network were annotated with functional gens sets associated with colorectal cancer and the mechanism of tumor progression and invasion. Our work also provides a simple-to-use nomogram 


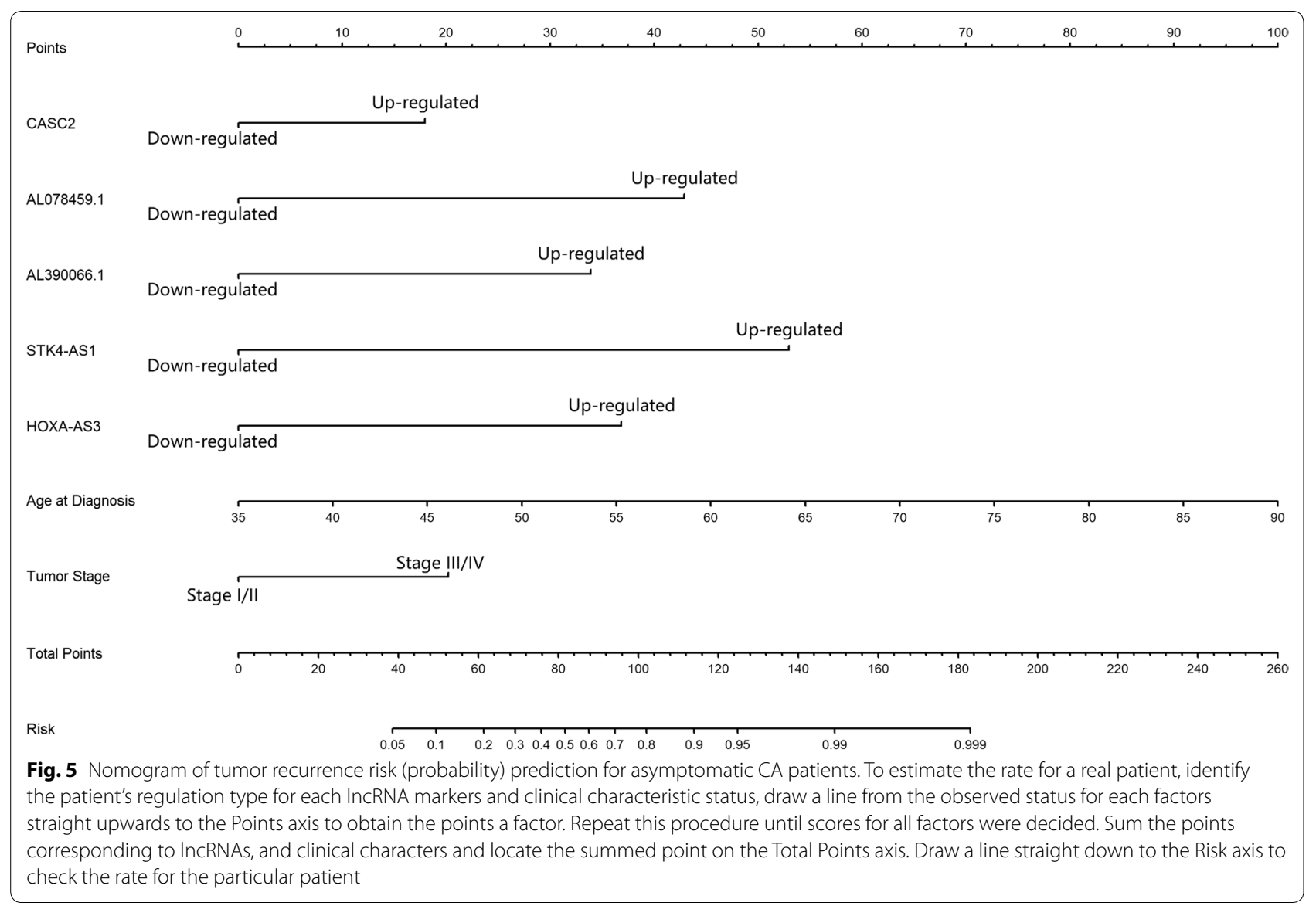

predicting the tumor recurrence risk for asymptotic CA patients based on the lncRNA markers identified in this study with clinical covariates. Though clinical validation is still needed, it is reasonable to conclude that these miRNAs are worthwhile for further study as novel candidate prognostic biomarkers for the survival of CA.

\section{Supplementary information}

Supplementary information accompanies this paper at https://doi. org/10.1186/s12967-019-2049-2.

Additional file 1. Differently expressed genes (IncRNAs, miRNAs and mRNAs) selected between CA patients with and without tumor recurrence.

\section{Abbreviations}

TCGA: The Cancer Genome Atlas; CA: colon adenocarcinoma; CRC: colorectal carcinoma; IncRNA: long non-coding RNA; ceRNA: competing endogenous RNA; KEGG: Kyoto Encyclopedia of Genes and Genomes; GO: Gene Ontology; BP: biological process; MF: molecular function; CC: cellular component.

\section{Authors' contributions}

$\mathrm{HY}$ is the principle investigator. FC and CD conducted statistical analysis and draft the manuscript. FC, ZL and CD conducted data management and bioinformatics analysis. HY and FC edited and revised the manuscript. All authors read and approved the final manuscript.

\section{Funding}

This study has been supported by the National Natural Science Foundation of China (Grant Number 81703325) and Research Support Program for Young Teachers in Xi'an Jiaotong University (Grant Number YX1 K0801001).

\section{Availability of data and materials}

The datasets analyzed during the current study are available at the TCGA database (https://cancergenome.nih.gov/).

Ethics approval and consent to participate

This study does not contain any work with human participants conducted by any of the authors.

\section{Consent for publication}

Not applicable.

\section{Competing interests}

The authors declare that they have no competing interests.

\section{Author details}

${ }^{1}$ Department of Epidemiology and Biostatistics, School of Public Health, Xi'an Jiaotong University Health Science Center, 76 Yanta Xilu Road, Xi'an 710061,

Shaanxi, China. ${ }^{2}$ First Affiliated Hospital of Xi'an Jiaotong University, 277 Yanta 
Xilu Road, Xi'an 710061, Shaanxi, China. ${ }^{3}$ Department of Preventive Medicine, Shantou University Medical College, 22 Xinling Road, Jinping District, Shantou 515041, Guangdong, China.

Received: 7 March 2019 Accepted: 25 August 2019 Published online: 30 August 2019

\section{References}

1. Siegel RL, et al. Cancer statistics, 2017. CA Cancer J Clin. 2017;67(1):7-30.

2. Mcmillan $D$, Wotherspoon $H$, Fearon $K$, et al. A prospective study of tumor recurrence and the acute-phase response after apparently curative colorectal cancer surgery. Am J Surg. 1995;170(4):319.

3. Kazushige $\mathrm{K}$, Hiroaki N, Keisuke $\mathrm{H}$, et al. Nomogram predicting survival after recurrence in patients with stage I to III colon cancer. Dis Colon Rectum. 2018;61(9):1053-62.

4. Usher-Smith JA, Walter FM, Emery JD, Win AK, Griffin SJ. Risk prediction models for colorectal cancer: a systematic review. Cancer Prev Res (Phila). 2016;9:13-26.

5. Bu D, Yu K, Sun S, Xie C, Skogerbo G, Miao R, et al. Non-code v3.0: integrative annotation of long noncoding RNAs. Nucleic Acids Res. 2012;40:210-5.

6. Yang G, Lu X, Yuan L. LncRNA: A link between RNA and cancer. Biochimica et Biophysica Acta (BBA) Gene Regul Mech. 2014;1839(11):1097-109.

7. Shi X, Ma C, Zhu Q, Yuan D, et al. Upregulation of long intergenic noncoding RNA 00673 promotes tumor proliferation via LSD1 interaction and repression of NCALD in non-small-cell lung cancer. Oncotarget. 2016;7:25558-75

8. Wang P, Liu B, Xia Y, et al. Long noncoding RNA-low expression in tumor inhibits the invasion and metastasis of esophageal squamous cell carcinoma by regulating p53 expression. Mol Med Rep. 2016;13:3074-82.

9. Shang C, Guo Y, Zhang J, et al. Silence of long noncoding RNA UCA1 inhibits malignant proliferation and chemotherapy resistance to adriamycin in gastric cancer. Cancer Chemother Pharmacol. 2016;77:1061-7.

10. Zhang D, Cao C, Liu L, et al. Up-regulation of LncRNA SNHG20 predicts poor prognosis in hepatocellular carcinoma. J Cancer. 2016;7:608-17.

11. Bian Z, Jin L, Zhang J, et al. LncRNA-UCA1 enhances cell proliferation and 5-fluorouracil resistance in colorectal cancer by inhibiting miR-204-5p. Sci Rep. 2016;6:23892.

12. Zhang Z, Qian W, Wang S, et al. Analysis of IncRNA-associated ceRNA network reveals potential IncRNA biomarkers in human colon adenocarcinoma. Cell Physiol Biochem. 2018;49(5):1778-91.

13. Yue B, Qiu S, Zhao S, et al. LncRNA-ATB mediated E-cadherin repression promotes the progression of colon cancer and predicts poor prognosis. J Gastroenterol Hepatol. 2016;31(3):595-603.

14. Ye Z, Zhou M, Tian B, et al. Expression of IncRNA-CCAT1, E-cadherin and $\mathrm{N}$-cadherin in colorectal cancer and its clinical significance. Int J Clin Exp Med. 2015;8(3):3707.

15. Ghosal S, Das S, Sen R, et al. HumanViCe: host ceRNA network in virus infected cells in human. Front Genet. 2014;5:249.

16. Poliseno L, Pandolfi P. PTEN ceRNA networks in human cancer. Methods. 2015;77-78:41-50

17. Sui J, Li Y, Zhang Y, et al. Integrated analysis of long non-coding RNAassociated ceRNA network reveals potential IncRNA biomarkers in human lung adenocarcinoma. Int J Oncol. 2016;49(5):2023.

18. Zhang Z, Wang S, Ji D, et al. Construction of a ceRNA network reveals potential IncRNA biomarkers in rectal adenocarcinoma. Oncol Rep. 2018;39(5):2101-13.

19. Zhai X, Xue Q, Liu Q, et al. Colon cancer recurrence-associated genes revealed by WGCNA co-expression network analysis. Mol Med Rep. 2017; 16(5):6499-505.

20. Meng Z, Long $\mathrm{H}$, Zicheng Z, et al. Recurrence-Associated Long Noncoding RNA Signature for Determining the Risk of Recurrence in Patients with Colon Cancer. Mol Ther Nucleic Acids. 2018;12:518-29.

21. Dai W, Feng $Y, M o ~ S$, et al. Transcriptome profiling reveals an integrated mRNA-IncRNA signature with predictive value of early relapse in colon cancer. Carcinogenesis. 2018:39(10):1235-44.

22. Zhang T, Wu D, Deng S, et al. Integrated analysis reveals that long non-coding RNA TUBA4B can be used as a prognostic biomarker in various cancers. Cell Physiol Biochem. 2018;49(2):530-44. https://doi. org/10.1159/000492991.

23. Benjamini Y, Hochberg Y. Controlling the false discovery rate: a practical and powerful approach to multiple hypothesis testing. J R Stat Soc B. 1995:57:289-300.

24. Jeggari A, Marks D, Larsson E. miRcode: a map of putative microRNA target sites in the long non-coding transcriptome. Bioinformatics. 2012;28(15):2062-3.

25. Li J, Liu S, Zhou H, et al. Starbase v2.0: decoding miRNA-ceRNA, miRNAncRNA and protein-RNA interaction networks from large-scale clip-seq data. Nucleic Acids Res. 2013. https://doi.org/10.1093/nar/gkt1248.

26. Kozomara A, Griffiths-Jones S. miRBase: annotating high confidence microRNAs using deep sequencing data. Nucleic Acids Res. 2014;42:68-73.

27. Shannon P, Markiel A, Ozier O, et al. Cytoscape: a software environment for integrated models of biomolecular interaction networks. Genome Res. 2003;13:2498-504.

28. Morris $\mathrm{JH}$, Apeltsin L, Newman AM, et al. ClusterMaker: a multi-algorithm clustering plugin for Cytoscape. BMC Bioinform. 2011;12(1):436.

29. Bindea G, Mlecnik B, Hackl H, et al. ClueGO: a Cytoscape plug-in to decipher functionally grouped gene ontology and pathway annotation networks. Bioinformatics. 2009;25(8):1091-3.

30. Ashburner M, Ball C, Blake J, et al. Gene Ontology: tool for the unification of biology. Nat Genet. 2000;25:25-9.

31. Altermann E, et al. PathwayVoyager: pathway mapping using the Kyoto Encyclopedia of Genes and Genomes (KEGG) database. BMC Genom. 2005;6:60. https://doi.org/10.1186/1471-2164-6-60.

32. Croft D, Mundo A, Haw R, et al. The Reactome pathway Knowledgebase. Nucleic Acids Res. 2013;42(D1):D649-55.

33. Mosallaei M. Evaluation of miR-21 inhibition and its impact on cancer susceptibility candidate 2 long noncoding RNA in colorectal cancer cell line. Adv Biomed Res. 2018. https://doi.org/10.4103/abr.abr_214_16.

34. Xin Y, Heyi Z, Gary T, et al. CASC2: an emerging tumour-suppressing long noncoding RNA in human cancers and melanoma. Cell Prolif. 2018. https ://doi.org/10.1111/cpr.12506.

35. Huang G, Wu X, Li S, et al. The long noncoding RNA CASC2 functions as a competing endogenous RNA by sponging miR-18a in colorectal cancer. Scientific Rep. 2016;6:26524. https://doi.org/10.1038/srep26524.

36. Lee Y, Yang C, Hsu T, et al. Abstract 1146: STK4 downregulation promotes tumor invasion/migration and is associated with poor prognosis in human colon cancer. Can Res. 2013;73:1146-1146.

37. Wu F, Zhang C, Cai J, et al. Upregulation of long noncoding RNA HOXAAS3 promotes tumor progression and predicts poor prognosis in glioma. Oncotarget. 2017:8(32):53110.

38. Stanilov N, Miteva L, Deliysky T, et al. Advanced colorectal cancer is associated with enhanced IL-23 and IL-10 serum levels. Lab Med. 2010;41(3):159-63.

39. Wei J, Liu H, Zhu X, et al. The expression and significance of IL-10 and IL-12 in colorectal cancer. Mod Prev Med. 2014;16:3014-6.

40. Nosho K, Baba Y, Tanaka N, et al. Tumour-infiltrating T-cell subsets, molecular changes in colorectal cancer, and prognosis: cohort study and literature review. J Pathol. 2010;222(4):350-66.

41. Smyth M, Teng M, Swann J, et al. CD4+CD25+T regulatory cells suppress NK cell-mediated immunotherapy of cancer. J Immunol. 2006;176(3):1582-7.

42. Kajihara M, Takakura K, Kanai T, et al. Dendritic cell-based cancer immunotherapy for colorectal cancer. World J Gastroenterol. 2016;22(17):4275-86.

43. Yomoda T, Sudo T, Kawahara A, et al. The immunoscore is a superior prognostic tool in stages II and III colorectal cancer and is significantly correlated with programmed death-ligand 1 (PD-L1) expression on tumor-infiltrating mononuclear cells. Ann Surg Oncol. 2019;26(2):415-24.

44. Nielsen $\mathrm{H}$, Hansen U, Christensen I, et al. Independent prognostic value of eosinophil and mast cell infiltration in colorectal cancer tissue. J Pathol. 2015:189(4):487-95.

45. Ohtsukasa S, Okabe S, Yamashita H, et al. Increased expression of CEA and MHC class I in colorectal cancer cell lines exposed to chemotherapy drugs. J Cancer Res Clin Oncol. 2003;129(12):719-26.

46. Karlsson R, Pedersen E, Wang Z, et al. Rho GTPase function in tumorigenesis. BBA Rev Cancer. 2009;1796(2):91-8. 
47. Pang X, Rui L, Shi D, et al. Knockdown of Rhotekin 2 expression suppresses proliferation and induces apoptosis in colon cancer cells. Oncol Lett. 2017;14(6):8028-34.

48. Stolfi C, Rizzo A, Franzè E, et al. Involvement of interleukin-21 in the regulation of colitis-associated colon cancer. J Exp Med. 2011;208(11):2279-90.

49. Sim G, Radvanyi L. The IL-2 cytokine family in cancer immunotherapy. Cytokine Growth Factor Rev. 2014;25(4):377-90.

50. Shibutani M, Maeda K, Nagahara H, et al. Tumor-infiltrating lymphocytes predict the chemotherapeutic outcomes in patients with stage IV colorectal cancer. Vivo. 2018;32:151-8.

51. Udall M, Rizzo M, Kenny J, et al. PD-L1 diagnostic tests: a systematic literature review of scoring algorithms and test-validation metrics. Diagn Pathol. 2018;13:12. https://doi.org/10.1186/s13000-018-0689-9.

52. Straus D. TNFa and IL-17 cooperatively stimulate glucose metabolism and growth factor production in human colorectal cancer cells. Mol Cancer. 2013;12(1):1-13.

53. Todaro M, Perez A, Scopelliti A, et al. IL-4-mediated drug resistance in colon cancer stem cells. Cell Cycle. 2008;7(3):309-13.

54. Li Z, Lin F, Fan $\mathrm{H}$, et al. The anticancer effect of serum thymic factor 9 peptide on human colon cancer HT-29. Carcinog Teratog Mutagen. 2011;23(5):374-6.

55. Wang L, Wang Y, Song Z, et al. Deficiency of interferon-gamma or its receptor promotes colorectal cancer development. J Interferon Cytokine Res. 2015;35(4):273-80.
56. Goto K, Zako K, Komizu Y, et al. Inhibitory effects of hybrid liposomes composed of phosphatidylcholine and docosahexaenoic acid on the growth of colon cancer cells along with apoptosis and differentiation. Chem Lett. 2010;40(1):90-2.

57. Chen $Y, Y u X, X u Y$, et al. Identification of dysregulated IncRNAs profiling and metastasis-associated IncRNAs in colorectal cancer by genome-wide analysis. Cancer Med. 2017;6(10):2321-30.

58. Zhou Q, Hou Z, Zuo S, et al. LUCAT1 promotes colorectal cancer tumorigenesis by targeting the ribosomal protein L40-MDM2-p53 pathway through binding with UBA52. Cancer Sci. 2019;110(4):1194-207.

59. Yu H, Wang C, Yuan Z, et al. LncRNA NEAT1 promotes the tumorigenesis of colorectal cancer by sponging miR-193a-3p. Cell Prolif. 2019;52(1):e12526. https://doi.org/10.1111/cpr.12526.

60. Ge H, Yan Y, Wu D, et al. Potential role of LINC00996 in colorectal cancer: a study based on data mining and bioinformatics. Onco Targets Ther. 2018;11:4845-55.

\section{Publisher's Note}

Springer Nature remains neutral with regard to jurisdictional claims in published maps and institutional affiliations.
Ready to submit your research? Choose BMC and benefit from:

- fast, convenient online submission

- thorough peer review by experienced researchers in your field

- rapid publication on acceptance

- support for research data, including large and complex data types

- gold Open Access which fosters wider collaboration and increased citations

- maximum visibility for your research: over $100 \mathrm{M}$ website views per year

At BMC, research is always in progress.

Learn more biomedcentral.com/submissions 